\title{
Whole exome sequencing of thymic neuroendocrine tumor with ectopic ACTH syndrome
}

\author{
Yanli Li',*, Ying Peng1,*, Xiuli Jiang', Yulong Cheng ${ }^{3}$, Weiwei Zhou', Tingwei Su', \\ Jing Xie ${ }^{2}$, Xu Zhong' ${ }^{1}$, Dalong Song', Luming Wu' ${ }^{1}$, Liwen Fan', Min Li', Jie Hong', \\ Weiqing Wang', Guang Ning ${ }^{1,3}$ and Yanan Cao'
}

\begin{abstract}
'Shanghai Clinical Center for Endocrine and Metabolic Diseases, Shanghai Key Laboratory for Endocrine Tumors and ${ }^{2}$ Department of Pathology, Rui-Jin Hospital, Shanghai Jiao-Tong University School of Medicine, Shanghai, China, and 'Laboratory of Endocrinology and Metabolism, Institute of Health Sciences, Shanghai Institutes for Biological Sciences (SIBS), Chinese Academy of Sciences (CAS) \& Shanghai Jiao-Tong University School of Medicine (SJTUSM), Shanghai, China *(Y Li and Y Peng contributed equally to this work)
\end{abstract}

Correspondence should be addressed to $\mathrm{Y}$ Cao Email

caoyanan@vip.sina.com

\begin{abstract}
Objective: Thymic neuroendocrine tumor is the second-most prevalent cause of ectopic adrenocorticotropic hormone (ACTH) syndrome (EAS), which is a rare disease characterized by ectopic ACTH oversecretion from nonpituitary tumors. However, the genetic abnormalities of thymic neuroendocrine tumors with EAS remain largely unknown. We aim to elucidate the genetic abnormalities and identify the somatic mutations of potential tumor-related genes of thymic neuroendocrine tumors with EAS by whole exome sequencing.

Design and methods: Nine patients with thymic neuroendocrine tumors with EAS who were diagnosed at Shanghai Clinical Center for Endocrine and Metabolic Diseases in Ruijin Hospital between 2002 and 2014 were enrolled.

We performed whole exome sequencing on the DNA obtained from thymic neuroendocrine tumors and matched peripheral blood using the Hiseq2000 platform.

Results: We identified a total of 137 somatic mutations (median of 15.2 per tumor; range, 1-24) with 129 singlenucleotide mutations (SNVs). The predominant substitution in these mutations was C:G > T:A transition. Approximately $80 \%$ of detected mutations resulted in amino acid changes. However, we failed to discover any recurrent mutations in these nine patients. By functional predictions, HRAS, PAK1 and MEN1, previously reported in neuroendocrine tumors, were identified as candidate tumor-related genes associated with thymic neuroendocrine tumors.

Conclusions: Using whole exome sequencing, we identified genetic abnormalities in thymic neuroendocrine tumors with EAS. Thereby, this study acts as a further supplement of the genetic features of neuroendocrine tumors. Somatic mutations of three potential tumor-related genes (HRAS, PAK1 and MEN1) might contribute to the tumorigenesis of thymic neuroendocrine tumors with EAS.
\end{abstract}

\section{Introduction}

Ectopic adrenocorticotropic hormone (ACTH) syndrome (EAS) accounts for $12 \%-17 \%$ cases of Cushing's syndrome (1), whose diverse clinical manifestations such as moon face, hypokalemia and hypertension are due to chronic

www.eje-online.org DOI: 10.1530/EJE-16-0546
() 2017 European Society of Endocrinology Printed in Great Britain exposure to excess glucocorticoids. EAS is characterized by ACTH hypersecretion from nonpituitary tumors such as small-cell lung tumor, bronchus carcinoid, thymic neuroendocrine tumor and other neuroendocrine 
tumors. Among these, thymic neuroendocrine tumors, characterized by aggressive and poor prognosis due to local recurrence and metastasis, is the second common cause of EAS (1). Surgery is the most common strategy for treating this tumor, whereas there are only less effective medical therapies due to the lack of knowledge on the molecular mechanisms of its tumorigenesis and metastasis. Therefore, better mechanistic studies on thymic neuroendocrine tumors are necessary to understand this disease and to identify the potential therapeutic targets.

By now, some progresses have already been made in the elucidation of the molecular mechanisms of thymic neuroendocrine tumors with EAS. Hypomethylation of the proopiomelanocortin (POMC) promoter region in thymic neuroendocrine tumors causes POMC overexpression, which is correlated with EAS (2). Meanwhile, by using cDNA microarray, dysregulation of Wnt and Notch signaling pathways in thymic neuroendocrine tumors with EAS has been reported by Bi et al. (3), indicating that these two signaling pathways may be involved in tumorigenesis or tumor development. However, the abnormalities of thymic neuroendocrine tumors at the genetic level have not yet been elucidated.

In the current study, we performed whole exome sequencing on nine patients with thymic neuroendocrine tumors with EAS and matched peripheral blood DNA. Thereby, a great number of genetic alterations were revealed and three mutated, candidate tumor-related genes were identified. These genetic abnormalities of thymic neuroendocrine tumors with EAS may provide valuable clues for further studies.

\section{Subjects and methods}

\section{Subjects and clinical data}

Nine patients with thymic neuroendocrine tumors with EAS were diagnosed at the Shanghai Clinical Center for Endocrine and Metabolic Diseases in Ruijin Hospital between 2002 and 2014 according to the following criteria (4): (1) typical clinical features of Cushing's syndrome such as moon face, hirsutism, hypokalemia, hypertension and diabetes; (2) biochemical abnormalities: elevated serum ACTH and cortisol, 24-h urinary free cortisol (UFC), failure to suppress serum cortisol as assessed by high-dose (administration of $8 \mathrm{mg}$ dexamethasone for 2 days) dexamethasone suppression test (HDDST); (3) image examinations: thoracic computed tomography (CT) or positron emission tomography (PET)-CT scanning revealing mediastinal tumors and (4) pathological examination of surgical specimen by two pathologists confirming the diagnosis of thymic neuroendocrine tumors. ACTH was positive by histological staining in

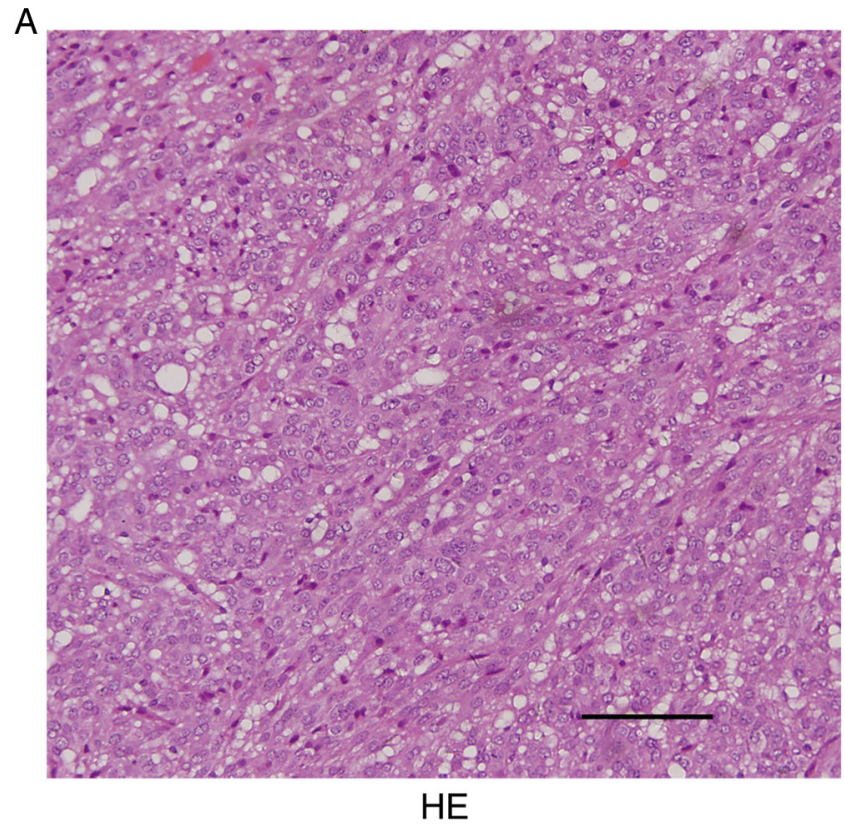

B

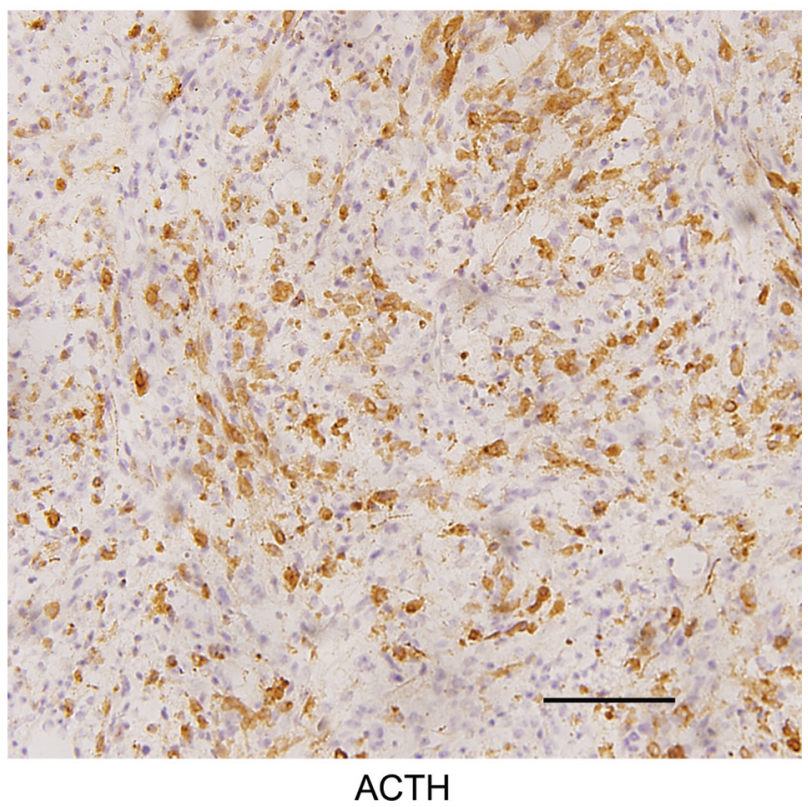

\section{Figure 1}

Representative histological micrographs of thymic neuroendocrine tumors with EAS. (A) Representative micrographs of hematoxylin-eosin (HE) staining in thymic neuroendocrine tumors. (B) Immunohistochemical staining for ACTH in thymic neuroendocrine tumors is shown.

Scale bar, $100 \mu \mathrm{m}$. 
all patients (Fig. 1 and Supplementary Fig. 1, see section on supplementary data given at the end of this article). Serum ACTH levels were measured using an ELSA-ACTH immunoradiometric assay (Cisbio Bioassays, Codolet, Languedoc, France). Serum cortisol and UFC were measured using Access Immunoassay Systems (Beckman Coulter Inc, Fullerton, CA, USA). Informed consent was obtained from all participants. All protocols were approved by the Rui-jin Hospital Ethics Committee, Shanghai JiaoTong University School of Medicine.

\section{DNA extraction and exome sequencing}

Genomic DNA was extracted from frozen thymic neuroendocrine tumors and matched blood samples using the QIAGEN DNeasy Blood \& Tissue Kit (Qiagen). Genomic DNA was isolated from tumors and matched peripheral blood was collected from nine patients of thymic neuroendocrine tumors with EAS. Extracted DNA was randomly cut into fragments of 200 to $300 \mathrm{bp}$ using Covaris technology, and then adapters were ligated to both ends of the resulting fragments. DNA was then amplified by ligation-mediated polymerase chain reaction (LM-PCR), purified and hybridized to the NimbleGenEZ $44 \mathrm{M}$ human exome array for enrichment, and nonhybridized fragments were then removed by washing. Captured LM-PCR products were subjected to quantitative PCR to estimate the magnitude of enrichment. Each captured library was then loaded on the Illumina Hiseq2000 platform (Illumina, San Diego, CA, USA) and sequenced independently to ensure that each sample met the desired average fold-coverage. Raw image files were processed using Illumina base calling Software 1.7 for base calling with default parameters, and the sequence of each individual was generated as $90 \mathrm{bp}$, paired-end reads.

\section{Exome capture reads mapping and variation detection}

Reads containing sequencing adapters were removed and low-quality reads containing more than five unknown bases and low base quality bases were discarded. Highquality reads were aligned to the NCBI human reference genome (hg19) by BWA (v0.5.9) using default parameters (5). Picard (v1.54) was used to mark duplicate reads, followed by GATK (v1.0.6076) (6) to improve the alignment accuracy. Potential somatic substitutions were predicted by Varscan2.2.5. Then, we used our in-house pipeline to identify somatic mutations with the following major criteria: (1) adjacent somatic mutation distance of
$>10 \mathrm{bp}$; (2) mapping quality significantly higher than 30 (Wilcoxon rank-sum test, $P<0.2$ ); (3) base quality significantly higher than 20 (Wilcoxon rank-sum test, $P<0.05) ;(4)$ statistically significant allele frequency changes between tumor and adjacent normal (Fisher's exact test, $P<0.05$ ); (5) no mutations in gap-aligned reads; (6) mutations significantly enriched within $5 \mathrm{bp}$ of $5^{\prime}$ or $3^{\prime}$ ends of the reads and not in simple repeat region. To obtain high confidence somatic indels, we developed our own pipeline as follows: (1) candidate somatic indels were predicted using GATK SomaticIndel Detector with default parameters; (2) local realignment was performed with combined normal and tumor samples; (3) germline mutations were filtered to identify somatic variants in tumors. The filtered somatic singlenucleotide mutations and indels with high confidence were annotated using ANNOVAR (released 2011-10-02) (7) and subjected to further analysis. Potential tumorrelated candidate mutations were confirmed using Sanger sequencing and the following primers were confirmed: HRAS: 5'-GGAGCGATGACGGAATATAA and 3'-TTGATCTGCTCCCTGTACTG; PAK1: 5'-GGGCACAGA GGAGGAGTAAT and 3'-GGCTTCTTCTTCTGCTTCTC; MEN1: 5'-TCATGCCTGGGTAGTGTTT and 3'-GTCCCAG GTCATAGAGCAG.

\section{Bioinformatics analysis and identification of potential tumor-related gene mutations}

Polyphen-2 (genetics.bwh.harvard.edu/pph2/) (8) and SIFT (sift.jcvi.org/) (9) were used to predict protein function by scoring somatic missense mutation. Moreover, we looked into damaging mutations (missense mutations, nonsense mutations, frameshift deletions or insertions (SIFT $<0.05$ or Polyphen $2>0.85$ )) in the Cancer Gene Census (http://cancer.sanger.ac.uk/census/) and The Human Gene Mutation Database at the Institute of Medical Genetics in Cardiff (www.hgmd.org). Sequences of candidate mutated genes were aligned using an online ClustalW tool to display the conservation of the mutated position in various species.

\section{Results}

\section{General clinical characteristics of the patients}

Nine patients of thymic neuroendocrine tumors with EAS, aged 27-64 years (median age: 47.6 years) were recruited, including four males and five females. The average level of preoperative serum ACTH was $398.6 \mathrm{pg} / \mathrm{mL}$ and that 
Table 1 Clinical characteristic of the nine thymic neuroendocrine tumors with EAS.

\begin{tabular}{|c|c|c|c|c|c|c|c|c|c|}
\hline \multirow[b]{2}{*}{ Characteristics } & \multicolumn{9}{|c|}{ Patient identification } \\
\hline & 1 & 2 & 3 & 4 & 5 & 6 & 7 & 8 & 9 \\
\hline Age (years) ${ }^{a}$ & 44 & 52 & 41 & 58 & 64 & 27 & 47 & 37 & 58 \\
\hline Gender & $\mathrm{F}$ & M & $\mathrm{F}$ & M & M & M & $\mathrm{F}$ & $\mathrm{F}$ & $\mathrm{F}$ \\
\hline Duration of history (m) & 24 & 2 & 12 & 24 & 3 & 1 & 5 & 2 & 3 \\
\hline \multicolumn{10}{|l|}{ Pre-op } \\
\hline Serum $A C T H^{b}$ & 413.8 & 282.8 & 65.3 & 476.8 & 176.6 & 312.6 & 365.8 & 92.3 & 1401 \\
\hline Serum cortisol $(08: 00 h)^{c}$ & 20.5 & 25.8 & 35.7 & 84.3 & 40.5 & 73.1 & 30.2 & 29.8 & 77.3 \\
\hline Serum cortisol (16:00h) & 31.4 & 23.7 & 31.5 & 78.7 & 32.9 & 46.7 & 29.7 & 35.1 & 74.6 \\
\hline Serum cortisol $(00: 00 \mathrm{~h})$ & 67.8 & 25.5 & 14.1 & 59.5 & 37.2 & 65.5 & 22 & 15.5 & 58 \\
\hline UFC $^{d}$ & 518 & 942.9 & 1044.9 & 1996.6 & 1667.7 & 1235.7 & 739.7 & 422.4 & 4793.4 \\
\hline HDDST (2nd day) UFC & 483.8 & 2231.5 & 1131.9 & 2041.5 & 1376.4 & 1383.9 & - & - & 5206.79 \\
\hline $\begin{array}{l}\text { HDDST (2nd day) serum } \\
\text { cortisol }\end{array}$ & 18 & 51.9 & 54.9 & 85 & 32.4 & 47.2 & - & - & 54.59 \\
\hline HDDST (3rd day) UFC & 213.7 & 1714.3 & 1138.3 & 2082.9 & 707.5 & 1511.5 & 252.7 & 475.1 & 5923.79 \\
\hline $\begin{array}{l}\text { HDDST (3rd day) serum } \\
\text { cortisol }\end{array}$ & 14.5 & 64.1 & 39.3 & 89.1 & 45.8 & 41.6 & 17.9 & 27.9 & 54.29 \\
\hline Tumor size $(\mathrm{cm})$ & 3.3 & 1 & 2.8 & 1.2 & 10 & 1.6 & 2.3 & 0.7 & 2 \\
\hline Metastasis & Lumbar & - & - & - & - & - & Lymphatic & - & - \\
\hline \multicolumn{10}{|l|}{ Post-op } \\
\hline Serum ACTH & 383.2 & 104.6 & 38.8 & 22.3 & 165 & 16.2 & 122.5 & 9.3 & 12.8 \\
\hline Serum cortisol $(08: 00 \mathrm{~h})$ & 17.7 & - & 13.2 & 5.9 & 46.6 & 8.7 & 8.6 & 6.6 & 6.2 \\
\hline Histological staining & + & + & + & + & + & + & + & + & + \\
\hline \multicolumn{10}{|l|}{ Follow-up (3 months) } \\
\hline Serum ACTH & 474 & 123.8 & 14.7 & 15.8 & - & 8.2 & 20.8 & 17.7 & 39.6 \\
\hline Serum cortisol (08:00 h) & 20.6 & 45.3 & 8.1 & 7.7 & - & 19.3 & 10.6 & 14.1 & 8.9 \\
\hline
\end{tabular}

${ }^{a}$ At the time of surgery; ${ }^{b}$ normal range $=12-78 \mathrm{pg} / \mathrm{mL}$; cserum cortisol $(\mu \mathrm{g} / \mathrm{dL}) ;{ }^{d} U F C$ : urinary free cortisol, normal range $=21-111 \mu \mathrm{g} / 24 \mathrm{~h}$.

$\mathrm{F}$, female; $\mathrm{M}$, male.

of UFC was $1484.6 \mu \mathrm{g} / 24 \mathrm{~h}$. Among these nine patients, patient 9 had the highest preoperative serum ACTH and UFC level. Thoracic CT or PET/CT scan detected a mediastinal mass $(0.7-10 \mathrm{~cm})$ in all patients. All the mediastinal masses were histologically confirmed as thymic neuroendocrine tumor after surgery (Fig. 1A). Immunohistochemically, the tumor cells were positive for ACTH in all patients (Fig. 1B). Two patients had lymphatic metastasis at first diagnosis. Although the tumor and metastatic lymph nodes were completely resected, patient 1 subsequently developed lumbar metastases at three-month follow-up. General clinical characteristics of the nine patients are summarized in Table 1.

\section{Overviews of tumor-specific mutated genes}

Whole exome sequencing was performed using the Hiseq2000 platform with NimbleGen 44M human exome array. The average sequencing depth on target was 228fold (200-285X), and 95.63\% (95.30-96.10\%) of the target regions were covered at least 10-fold (Fig. 2A, B and Supplementary Table 1). Germline mutations of MEN1 (multiple endocrine neoplasia type 1), which is associated with familial thymic neuroendocrine tumors, were explored. We identified two non-synonymous (p.T546A and p.G508D) and two synonymous (p.D423D and p.H438H) mutations. None of these mutations is a germline pathogenic variant according to the NCBI ClinVar database.

In total, 137 somatic mutations with a mean mutation rate of 15.2 per sample (range 1-24 mutations) were identified (Supplementary Table 2). These mutations consisted of 129 single-nucleotide mutations (SNVs) (90 nonsynonymous, 30 synonymous mutations, 8 stopgain and 1 splice-site mutation), 6 frameshift deletions, 1 frameshift insertion and 1 non-frameshift insertion (Supplementary Table 3). Approximately $80 \%$ of the mutations resulted in amino acid changes and $46 \%$ of the mutations were predicted to be damaging. Transitions were more common than transversions and the ratio of transitions versus transversions was 2.15:1. Predominant substitution in these mutations involved $\mathrm{C}: \mathrm{G}>\mathrm{T}$ :A transition, which is similar to most other neuroendocrine tumors (Fig. 2C and Supplementary Table 4). In addition, we analyzed the loss of heterozygosity ( $\mathrm{LOH})$ and copy number variation (CNV) from whole exome data. Depths and $\mathrm{B}$ allele frequencies (BAF) of four samples containing 
A

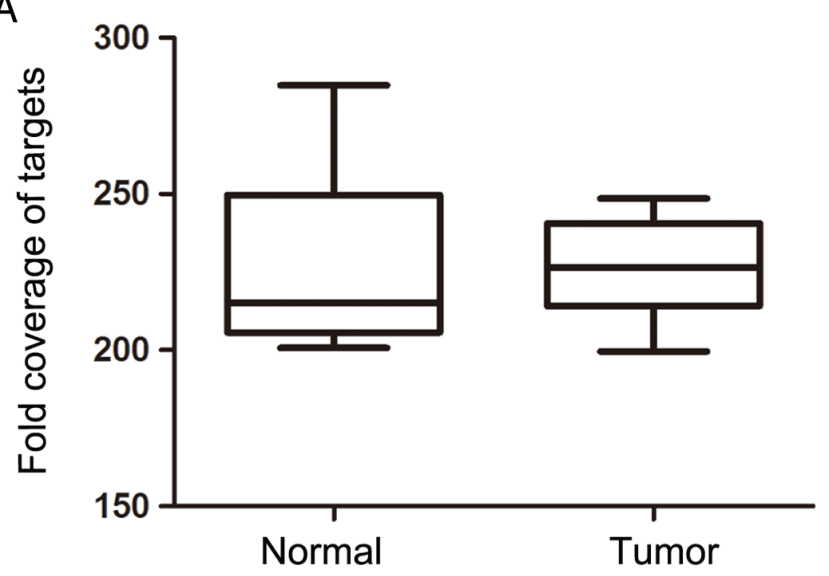

B

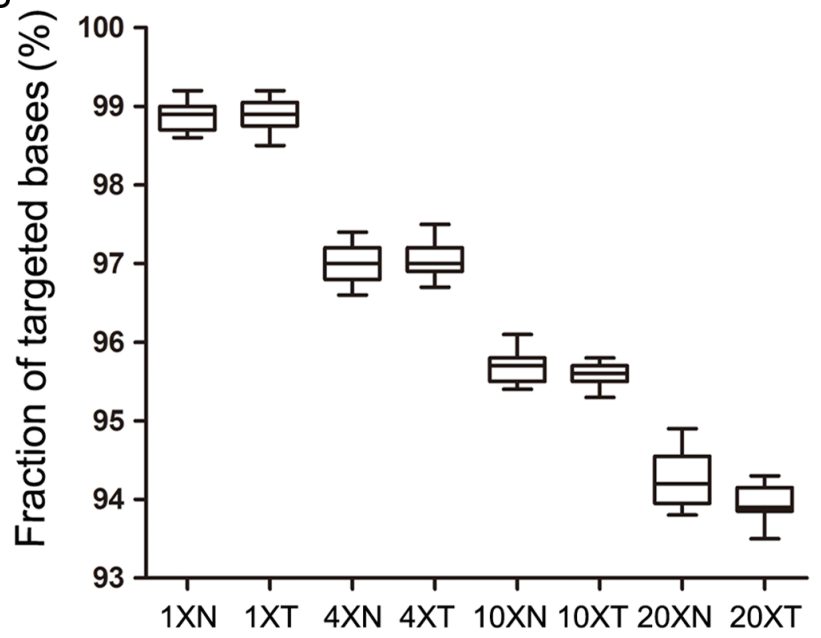

C

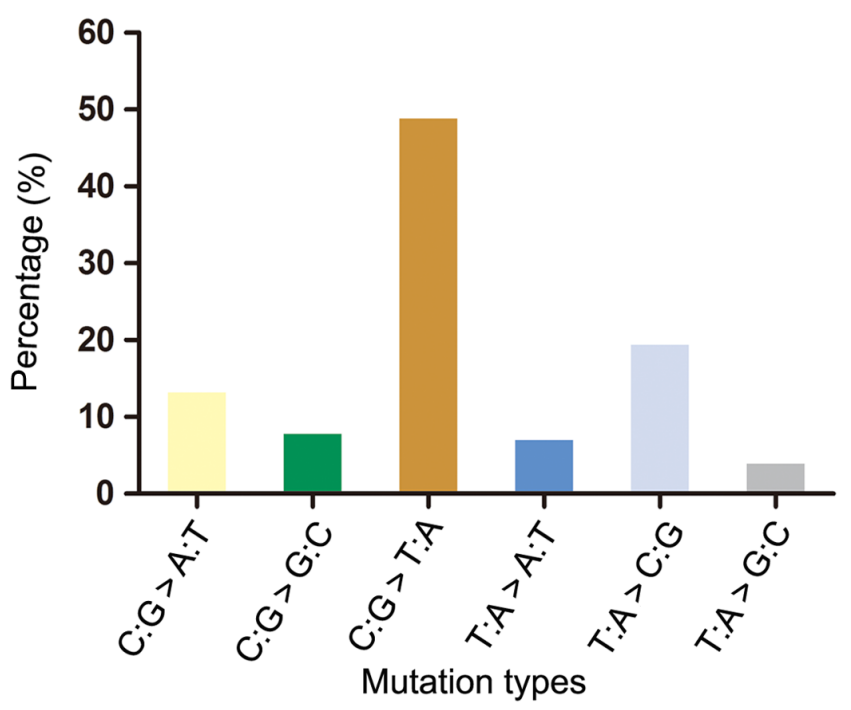

CNV or LOH are shown as Supplementary Fig. 3 and Supplementary Table 5. However, we failed to discover any recurrent mutations in our patient group.

\section{Identification and functional prediction of candidate mutated tumor-related genes}

To identify potential tumor-related candidate mutations in thymic neuroendocrine tumors with EAS, we screened 137 somatic mutations as follows: (1) mutations (frameshift indels, nonsense mutations and missense mutations) resulting in a deleterious effect on protein function predicted by two programs (Polyphen 2 and SIFT) and (2) mutated genes frequently reported to be involved in biological processes relevant to the tumorigenesis of other neuroendocrine tumors.

According to the previously mentioned criteria, three candidate genes (HRAS, PAK1 and MEN1), related to the cellular proliferation, were further analyze (Table 2). Harvey rat sarcoma viral oncogene homolog (HRAS), which belongs to the RAS family, acts as a transducer of signals in regulating diverse cellular processes such as proliferation, differentiation, migration and apoptosis. The somatic mutation of HRAS (p.Q61K), previously reported in thyroid cancer and pheochromocytoma (PCC) including metastatic PCCs $(10,11)$, was predicted to have a significantly deleterious effect on protein function. Furthermore, this position of HRAS (p.Q61) is evolutionarily conserved by the alignment of sequences. The patient who harbored the HRAS (p.Q61K) mutation

\section{Figure 2}

Fold-coverage of target regions for sequencing and spectrum of somatic point mutations in exon regions of thymic neuroendocrine tumors. (A) Box plots depict the distribution of fold-coverage of target regions from the whole exome sequencing data. Bold lines in the boxes represent the medians and the lines outside the boxes represent the first or third quartiles of fold-coverage. (B) Box plots show the distribution of the fraction of targeted bases covered by at least 1, 4, 10 and 20 reads across the nine samples. The bold lines in boxes represent the medians and the lines outside the boxes represent the first or third quartiles of fraction of targeted bases covered by reads. (C) The spectrum of somatic mutations in exome regions. The percentage of each mutation type was calculated in all nine thymic neuroendocrine tumors with EAS. The C:G-> T:A transition is the most common type of single-base substitution in thymic neuroendocrine tumors with EAS among the mutations studied. 
Table 2 Three potential tumor-related genes in thymic neuroendocrine tumors with EAS.

\begin{tabular}{|c|c|c|}
\hline Sample number & Gene & Mutation \\
\hline 1 & HRAS & Nonsynonymous \\
\hline 9 & PAK1 & Nonsynonymous \\
\hline 5 & MEN1 & Frameshift deletion \\
\hline
\end{tabular}

\begin{tabular}{l} 
CDS \\
\hline C. $181 C>A$ \\
c. $608 G>A$ \\
c. 690 delA \\
\hline
\end{tabular}

\begin{tabular}{l} 
AA \\
\hline p.Q61K \\
p.R203Q \\
p.G230fs \\
\hline
\end{tabular}

\begin{tabular}{c}
\hline SIFT \\
\hline 0.01 \\
0.01 \\
- \\
\hline
\end{tabular}

\begin{tabular}{c}
\hline Poly Phen2 \\
\hline 0.746 \\
0.612 \\
- \\
\hline
\end{tabular}

Damaging Damaging

$A A$, amino acid; CDS, coding sequence.

showed lumbar metastasis in the follow-up after three months. Somatic mutation (p.R203Q) of PAK1, located on chromosome 11 and encoding a serine/threonine kinase that is involved in many oncogenic signaling pathways, was found in patient 9, who showed the highest serum ACTH and UFC levels before surgery of all nine patients. This locus of PAK1 (p.R203) is also evolutionarily conserved and predicted to be damaging by Polyphen2 and SIFT.

MEN1, encoded by MEN1 (multiple endocrine neoplasia type 1) and containing three nuclear localization signals (NLSs) at the C-terminal of the protein, is located primarily in the nucleus and is more easily degraded in the cytoplasm (12). Remarkably, a frameshift deletion (c.690delA) of MEN1 was discovered by CT scan in patient 5 , who harbored the largest thymic tumor among the nine patients. This c.690delA/p.G230fs mutation of MEN1, which involves exon 2 , results in a truncated protein with deletion of two NLS at the C-terminal end. Therefore, this truncated menin is located in the cytoplasm and is more rapidly degraded (12). In addition, the locus mutation of MEN1 (c.690delA/p.G230fs) (Gly230 deletion, accession number: NM_000244) detected in thymic neuroendocrine tumors with EAS in this study, had not been previously reported (The Human Gene Mutation Database at the Institute of Medical Genetics in Cardiff and Cancer Gene Census) and hence represents a novel mutation.

\section{Discussion}

In our study, whole exome sequencing was performed on nine thymic neuroendocrine tumors with EAS. Our investigation identified a total of 137 somatic mutations, resulting in a mean value of 15.2 somatic mutations per tumor. However, we failed to discover any recurrent mutated genes in this patient group.

Furthermore, we identified three potentially relevant mutations (HRAS, PAK1 and MEN1) that may be associated with the tumorigenesis of thymic neuroendocrine tumors on the basis of functional predictions. HRAS, which belongs to the RAS oncogene family, plays an important role in RAS/RAF/ERK signaling pathway relevant to tumorigenesis and metastasis. Somatic mutation of HRAS has been identified in inverted urothelial papillomas (IUP) (13), thyroid cancer (11) and PCC and paraganglioma (PGL) $(10,14)$. Currás-Freixes et al. demonstrated that HRAS had the highest frequency (15.3\%) among mutated genes in their 98 PCCs and PGLs tumor samples. Importantly, this gene mutation occurred in one metastatic PGL (14). Additionally, Crona et al. reported $6.9 \%$ of somatic mutated HRAS (G13R, Q61K and Q61R) in PCCs (10). Moreover, they revealed that this position mutation of HRAS (p.Q61K) resulted in significant overexpression of phospho-ERK and activation of RAS/RAF/ERK pathway, which is responsible for proliferation and metastasis (10). In our study, patient 1 , who showed mediastinal lymph node metastasis at surgery and lumbar metastasis at three months after surgery, harbored this HRAS (p.Q61K) mutation. The previously described mutational hot spot for HRAS (p.Q61), conserved in three RAS isoforms (KRAS, $N R A S$ and HRAS), is localized in the nucleotide-binding region. We speculated that the somatic mutation of HRAS was the principal mutation associated with tumorigenesis of thymic neuroendocrine tumors and may be associated with lumbar metastasis. Selumetinib, tested in KRASmutant advanced non-small-cell lung cancer (NSCLC) in a phase 2 clinical trial, is a potent and selective MEK1/2 inhibitor of the RAS/RAF/MEK/ERK pathway (15). Interestingly, our results showed HRAS mutation in one patient with thymic neuroendocrine tumor, which could be treated in the future with selumetinib, targeting the RAS/RAF/MEK/ERK pathway.

p21 protein-activated kinases (PAKs) were a family of conserved serine/threonine kinases that regulate several cellular activities, including cytoskeletal dynamics, actin depolymerization, cell proliferation and invasion. PAK1, the most established member of the PAKs family, is overexpressed in several types of human tumors such as breast cancer and ovarian carcinoma $(16,17)$. Previously, Liu et al. reported that another member of the PAKs family, PAK3, was mostly upregulated in thymic neuroendocrine tumors with EAS and promoted cell invasiveness as well as migration mediated by the JNK pathway (18). Both PAK1 and 
PAK3 belong to group I PAKs and are evolutionarily conserved. PAK1 contains $90 \%$ homology at the kinase domain with PAK3. The p.R203Q mutation in PAK1 appears in the motif for PKA-interacting exchange (PIX) factor binding and is predicted to be deleterious to protein function. We believe that somatic mutation of PAK1 may contribute significantly to the development of this thymic tumor. Additionally, we demonstrated for the first time that somatic mutation of PAK1 occurs in thymic neuroendocrine tumors with EAS.

MEN1 gene encodes the nuclear protein menin, which contains two nuclear localization signals (NLS) at the C-terminal. MEN1 participates in the regulation of several cell functions such as gene transcription and cell proliferation, whereas it also acts as a tumor suppressor. Loss of heterozygosity (LOH) of MEN1 is the main cause of multiple endocrine neoplasia type 1 (MEN1) syndrome. Previously, approximately $25 \%$ of reported thymic neuroendocrine tumors were reported in MEN1 syndrome patients (19). Thymic neuroendocrine tumors occur in approximately $3.1-8 \%$ cases of MEN1 syndrome (20). Sometimes thymic neuroendocrine tumor is the first presentation in MEN1 syndrome. A recent genetic study has revealed that the somatic mutation of MEN1 occurs in sporadic pancreatic neuroendocrine tumors. However, the MEN1 somatic mutation has not been reported in thymic neuroendocrine tumors with EAS that are not associated with MEN1 syndrome. In our study, a novel somatic mutation of MEN1 (c.690delA) was identified and predicted to result in a truncated menin, with deletion of the NLS. This truncated menin is located in the cytoplasm and is therefore, more rapidly degraded (12). Thus, loss of menin in the nucleus results in cell proliferation and gene transcription. Moreover, this patient with a somatic mutation of MEN1 showed the largest tumor size $(10 \mathrm{~cm})$, which we speculate contributed significantly to the mutation of MEN1. To our knowledge, this is the first report regarding thymic neuroendocrine tumors with EAS harboring the somatic frameshift mutation of MEN1. This somatic, mutated MEN1 is a candidate mutation, probably responsible for tumorigenesis. However, potential functional confirmation of these mutated genes should been performed in a thymic cell line.

In conclusion, we revealed genetic alterations of thymic neuroendocrine tumors with EAS by whole exome sequencing. Somatic mutations of several genes including HRAS, PAK1 and MEN1 are presented for the first time, in thymic neuroendocrine tumors with EAS. This study indicates certain somatic mutations as potential tumor-related genes in thymic neuroendocrine tumors with EAS and provides scope for further functional studies on causative mutations of thymic neuroendocrine tumors with EAS.

\section{Supplementary data}

This is linked to the online version of the paper at http://dx.doi.org/10.1530/ EJE-16-0546.

\section{Declaration of interest}

The authors declare that there is no conflict of interest that could be perceived as prejudicing the impartiality of the research reported.

\section{Funding}

This work was supported by Natural Science Foundation of China (grants number 81390350 and 81400779) and the Key Laboratory for Endocrine and Metabolic Disease of Ministry of Health (grants number 1994DP131044).

\section{Author contribution statement}

Y Li and Y Peng performed the experiments; $Y$ Cao conceived and designed all the experiments; G Ning, W Wang and J Hong directed this work and edited the manuscript; Y Li, W Zhou, T Su, J Xie, Y Peng, X Zhong, L Wu, $M \mathrm{Li}, \mathrm{D}$ Song and L Fan collected the specimens and analyzed the data; $Y \mathrm{Li}, \mathrm{X}$ Jiang and $\mathrm{Y}$ Cheng wrote the manuscript and all the authors agreed on the final manuscript.

\section{References}

1 Isidori AM \& Lenzi A. Ectopic ACTH syndrome. Arquivos Brasileiros de Endocrinologia e Metabologia 200751 1217-1225. (doi:10.1590/S000427302007000800007)

2 Ye L, Li X, Kong X, Wang W, Bi Y, Hu L, Cui B \& Ning G. Hypomethylation in the promoter region of POMC gene correlates with ectopic overexpression in thymic carcinoids. Journal of Endocrinology 2005185 337-343. (doi:10.1677/joe.1.05963)

3 Bi YF, Liu RX, Ye L, Fang H, Li XY, Wang WQ, Zhang J, Wang KK, Jiang L, Su TW et al. Gene expression profiles of thymic neuroendocrine tumors (carcinoids) with ectopic ACTH syndrome reveal novel molecular mechanism. Endocrine-Related Cancer 200916 1273-1282. (doi:10.1677/ERC-08-0325)

4 Lacroix A, Feelders RA, Stratakis CA \& Nieman LK. Cushing's syndrome. Lancet 2015386 913-927. (doi:10.1016/S01406736(14)61375-1)

5 Li H \& Durbin R. Fast and accurate short read alignment with Burrows-Wheeler transform. Bioinformatics 200925 1754-1760.(doi:10.1093/bioinformatics/btp324)

6 McKenna A, Hanna M, Banks E, Sivachenko A, Cibulskis K, Kernytsky A, Garimella K, Altshuler D, Gabriel S, Daly M et al. The Genome Analysis Toolkit: a MapReduce framework for analyzing next-generation DNA sequencing data. Genome Research 201020 1297-1303. (doi:10.1101/gr.107524.110)

7 Wang K, Li M \& Hakonarson H. ANNOVAR: functional annotation of genetic variants from high-throughput sequencing data. Nucleic Acids Research 201038 e164. (doi:10.1093/nar/gkq603)

8 Adzhubei IA, Schmidt S, Peshkin L, Ramensky VE, Gerasimova A, Bork P, Kondrashov AS \& Sunyaev SR. A method and server for predicting damaging missense mutations. Nature Methods 20107 248-249. (doi:10.1038/nmeth0410-248) 
9 Kumar P, Henikoff S \& Ng PC. Predicting the effects of coding nonsynonymous variants on protein function using the SIFT algorithm. Nature Protocols 20094 1073-1081. (doi:10.1038/nprot.2009.86)

10 Crona J, Delgado Verdugo A, Maharjan R, Stalberg P, Granberg D, Hellman P \& Bjorklund P. Somatic mutations in H-RAS in sporadic pheochromocytoma and paraganglioma identified by exome sequencing. Journal of Clinical Endocrinology and Metabolism 201398 E1266-E1271. (doi:10.1210/jc.2012-4257)

11 Schulten HJ, Al-Maghrabi J, Al-Ghamdi K, Salama S, Al-Muhayawi S, Chaudhary A, Hamour O, Abuzenadah A, Gari M \& Al-Qahtani M. Mutational screening of RET, HRAS, KRAS, NRAS, BRAF, AKT1, and CTNNB1 in medullary thyroid carcinoma. Anticancer Research 201131 $4179-4183$.

12 Cao Y, Liu R, Jiang X, Lu J, Jiang J, Zhang C, Li X \& Ning G. Nuclearcytoplasmic shuttling of menin regulates nuclear translocation of \{beta\}-catenin. Molecular and Cellular Biology 200929 5477-5487. (doi:10.1128/MCB.00335-09)

13 McDaniel AS, Zhai Y, Cho KR, Dhanasekaran SM, Montgomery JS, Palapattu G, Siddiqui J, Morgan T, Alva A, Weizer A et al. HRAS mutations are frequent in inverted urothelial neoplasms. Human Pathology 201445 1957-1965.(doi:10.1016/j.humpath.2014.06.003)

14 Curras-Freixes M, Inglada-Perez L, Mancikova V, Montero-Conde C, Leton R, Comino-Mendez I, Apellaniz-Ruiz M, Sanchez-Barroso L, Aguirre Sanchez-Covisa M, Alcazar V et al. Recommendations for somatic and germline genetic testing of single pheochromocytoma and paraganglioma based on findings from a series of 329 patients. Journal of Medical Genetics 201552 647-656. (doi:10.1136/ jmedgenet-2015-103218)
15 Wood K, Hensing T, Malik R \& Salgia R. Prognostic and predictive value in KRAS in non-small-cell lung cancer: a review. JAMA Oncology 20162 805-812. (doi:10.1001/jamaoncol.2016.0405)

16 Balasenthil S, Sahin AA, Barnes CJ, Wang RA, Pestell RG, Vadlamudi RK \& Kumar R. p21-activated kinase-1 signaling mediates cyclin D1 expression in mammary epithelial and cancer cells. Journal of Biological Chemistry 2004279 1422-1428. (doi:10.1074/jbc. M309937200)

17 Schraml P, Schwerdtfeger G, Burkhalter F, Raggi A, Schmidt D, Ruffalo T, King W, Wilber K, Mihatsch MJ \& Moch H. Combined array comparative genomic hybridization and tissue microarray analysis suggest PAK1 at 11q13.5-q14 as a critical oncogene target in ovarian carcinoma. American Journal of Pathology 2003163 985-992. (doi:10.1016/S0002-9440(10)63458-X)

18 Liu RX, Wang WQ, Ye L, Bi YF, Fang H, Cui B, Zhou WW, Dai M, Zhang J, Li XY et al. p21-activated kinase 3 is overexpressed in thymic neuroendocrine tumors (carcinoids) with ectopic ACTH syndrome and participates in cell migration. Endocrine 201038 38-47. (doi:10.1007/s12020-010-9324-6)

19 Teh BT. Thymic carcinoids in multiple endocrine neoplasia type 1. Journal of Internal Medicine 1998243 501-504. (doi:10.1046/j.13652796.1998.00329.x)

20 Ferolla P, Falchetti A, Filosso P, Tomassetti P, Tamburrano G, Avenia N, Daddi G, Puma F, Ribacchi R, Santeusanio F et al. Thymic neuroendocrine carcinoma (carcinoid) in multiple endocrine neoplasia type 1 syndrome: the Italian series. Journal of Clinical Endocrinology and Metabolism 200590 2603-2609. (doi:10.1210/ jc.2004-1155)

Received 24 June 2016

Revised version received 6 November 2016

Accepted 15 November 2016 